\title{
Dangers of home do-it-yourself
}

\section{Yu Zhen Lau, Yun Mei Lau and Gentle Wong*}

Frimley Park Hospital, Portsmouth Road, Surrey GU16 7UJ, UK

A 20-year-old fit and well woman was urgently referred to our department from accident and emergency for removal of foreign body. This occurred following an accidental aspiration of a $3 \mathrm{~cm}$ iron nail. This was gripped between her teeth whilst changing the ceiling lightbulb, when she laughed at a friend's joke. Aside from feeling understandably nervous, she was otherwise asymptomatic; she denied chest pain, breathlessness, and haemoptysis.

On examination, she was well with no evidence of foreign body impaction in the airway at bronchial level, this meant no wheeze, decreased breath sounds, or haemoptysis; at tracheal level, stridor or coughing; at laryngeal level, gagging associated with hoarseness, or cyanosis; or if it was impacted in the oesophagus, dysphagia, nausea and vomiting, saliva drooling.

The chest X-ray highlighted the radio-opaque foreign body in the right lower lobe (Figure 1). In adults, foreign bodies are more commonly located in the right bronchus because it is wider and more vertical than the left main bronchus. This was eventually removed with flexible bronchoscopy, through a rigid bronchoscope, under fluoroscopic guidance. The patient sustained a pneumothorax postoperatively (Figure 2), a not uncommon complication, but eventually made a successful recovery following chest drain insertion (Figure 3).

If left alone, severe complications can result from a long retained foreign body. These include non-resolving pneumonia, lung abscess, lung fibrosis, obstructive emphysema, recurrent haemoptysis, and bronchiectasis. Bronchiectasis is one of the most important complications that may necessitate a surgical resection (lobectomy/ limited segmentectomy) (Figure 4).
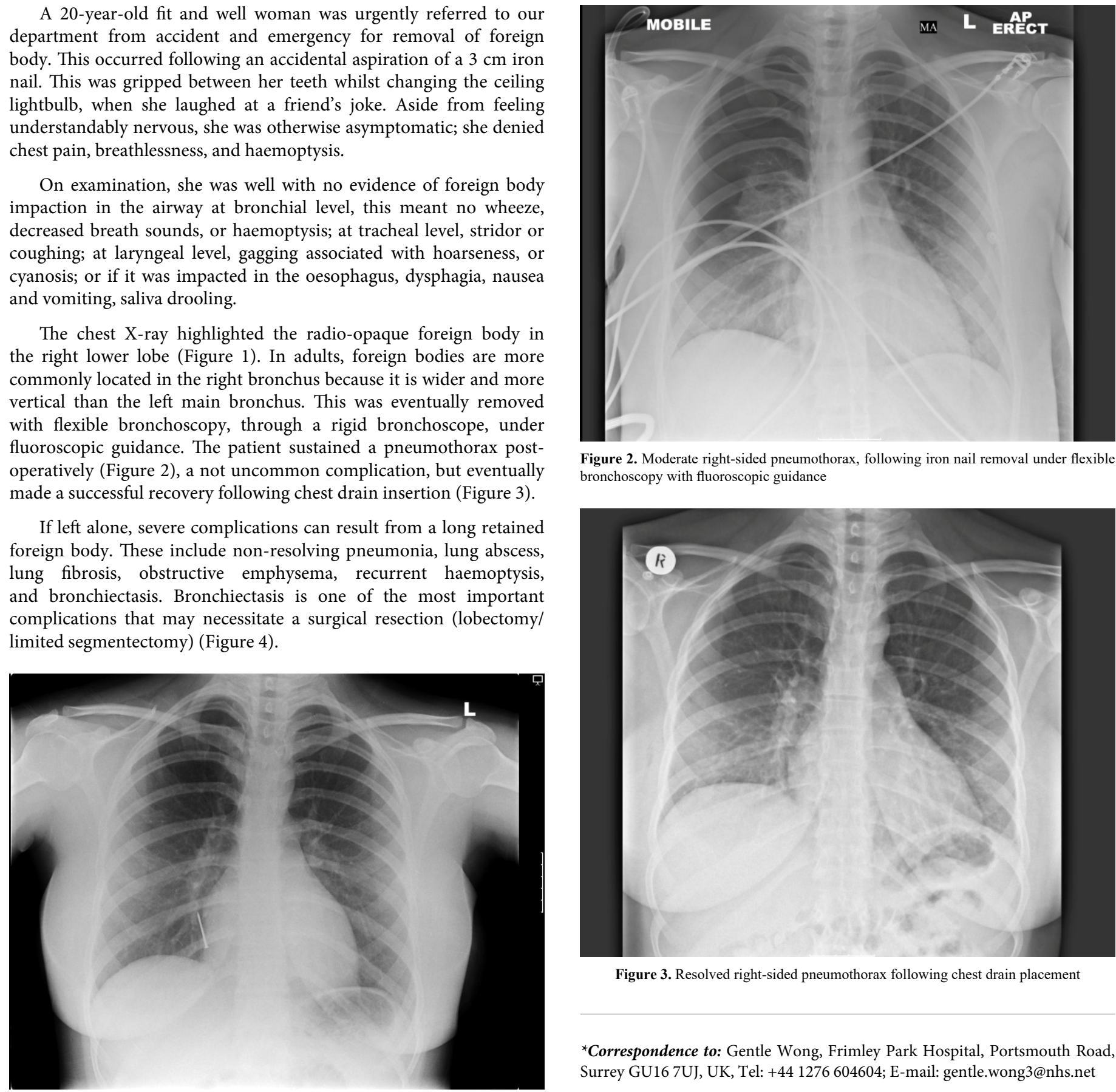

Figure 2. Moderate right-sided pneumothorax, following iron nail removal under flexible bronchoscopy with fluoroscopic guidance

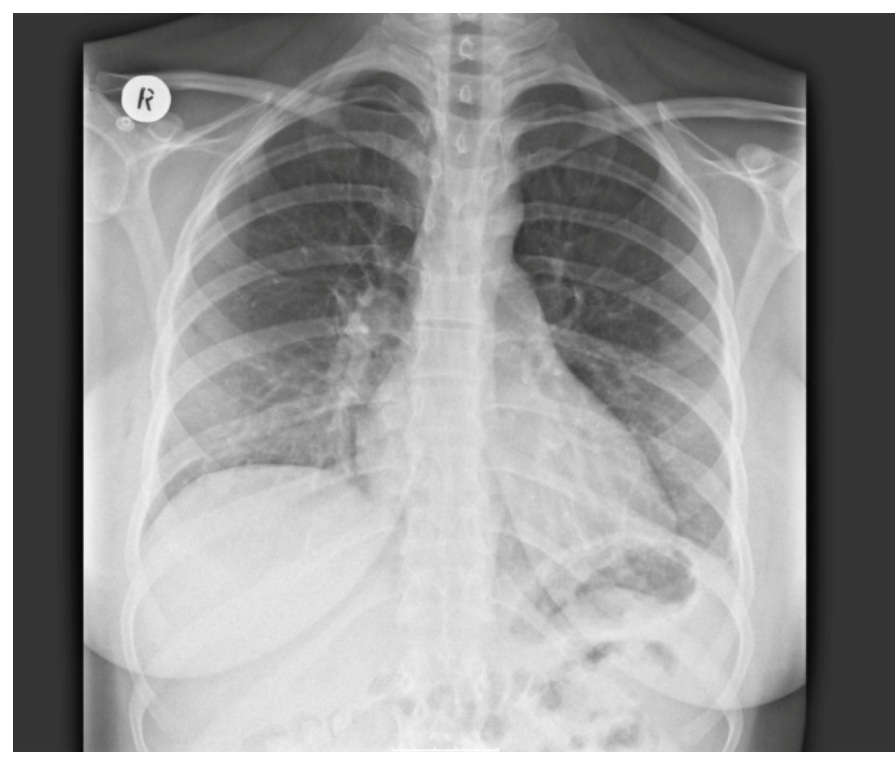

Figure 3. Resolved right-sided pneumothorax following chest drain placement

Figure 1. $3 \mathrm{~cm}$ radio-opaque nail clearly visible, projected over the right para-cardiac region, in the lower lobe bronchus

${ }^{*}$ Correspondence to: Gentle Wong, Frimley Park Hospital, Portsmouth Road, Surrey GU16 7UJ, UK, Tel: +44 1276 604604; E-mail: gentle.wong3@nhs.net

Received: August 09, 2019; Accepted: August 19, 2019; Published: August 21, 2019 


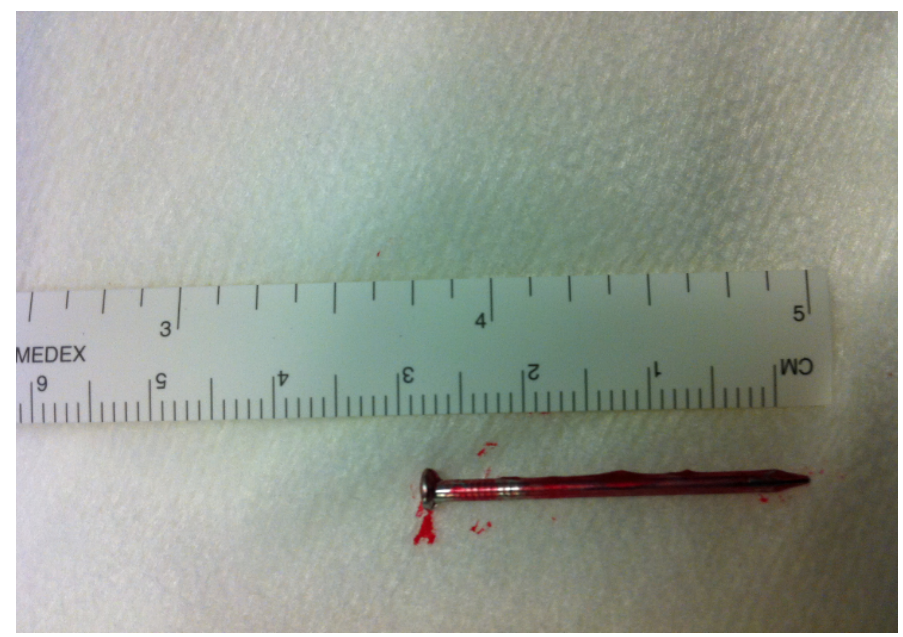

\section{Learning point}

1. Symptoms/signs may allow localisation of the inhaled/ingested foreign body.

2. Prompt specialist referral (either otolaryngology, cardiothoracic surgery, or respiratory physicians with a special interest in bronchoscopy) is important to minimise potential complications.

Figure 4. The $3 \mathrm{~cm}$ iron nail

Copyright: (O2019 Lau YZ. This is an open-access article distributed under the terms of the Creative Commons Attribution License, which permits unrestricted use, distribution, and reproduction in any medium, provided the original author and source are credited. 